\title{
MAJORIZATION ON A PARTIALLY ORDERED SET
}

\author{
F. K. HWANG
}

\begin{abstract}
We extend the classical concept of set majorization to the case where the set is partially ordered. We give a useful property which characterizes majorization on a partially ordered set. Quite unexpectedly, the proof of this property relies on a theorem of Shapley on convex games. We also give a theorem which is parallel to the Schur-Ostrowski theorem in comparing two sets of parameters in a function.
\end{abstract}

1. Introduction. The classical concept of majorization is defined on two $n$-sets of numbers $A=\left\{a_{1}, \ldots, a_{n}\right\}$ and $B=\left\{b_{1}, \ldots, b_{n}\right\}$ as follows. Let $a_{[i]}$ and $b_{[i]}$ denote the $i$ th largest numbers in $A$ and $B$, respectively. Then $A$ is said to majorize $B$ if and only if

$$
\sum_{i=1}^{k} a_{[i]}>\sum_{i=1}^{k} b_{[i]} \text { for } k=1, \ldots, n-1
$$

and

$$
\sum_{i=1}^{n} a_{[i]}=\sum_{i=1}^{n} b_{[i]}
$$

The concept of majorization is closely related to the concept of a Schur function. A function $f\left(x_{1}, \ldots, x_{n}\right)$ is called a Schur function [4] if, for all $i$ and $j$,

$$
\left(\frac{\partial f}{\partial x_{i}}-\frac{\partial f}{\partial x_{j}}\right)\left(x_{i}-x_{j}\right)>0 .
$$

The following theorem connects the two concepts:

THEOREM 1.1 (SCHUR [5], OSTROWSKI [4]). $f\left(a_{1}, \ldots, a_{n}\right)>f\left(b_{1}, \ldots, b_{n}\right)$ for all $A$ majorizing $B$ if and only if $f$ is a Schur function.

Set majorization can be naturally extended to vector majorization. We say that an $n$-vector $A=\left(a_{1}, \ldots, a_{n}\right)$ majorizes another $n$-vector $B=$ $\left(b_{1}, \ldots, b_{n}\right)$ if and only if

$$
\sum_{i=1}^{k} a_{i}>\sum_{i=1}^{k} b_{i} \text { for } k=1, \ldots, n-1
$$

and

$$
\sum_{i=1}^{n} a_{i}=\sum_{i=1}^{n} b_{i}
$$

Received by the editors September 1, 1978.

AMS (MOS) subject classifications (1970). Primary 06A10, 26A51, 26A86; Secondary 90D12. 
The concept of vector majorization has been proved useful in many instances ([1], [3]) where vector optimization is concerned.

Vector majorization can be interpreted in a different way which leads to a further extension. Let $P=\left\{p_{1}, \ldots, p_{n}\right\}$ be a set of points ordered linearly by " $\rightarrow$ " and let $A=\left\{a_{1}, \ldots, a_{n}\right\}$ and $B=\left\{b_{1}, \ldots, b_{n}\right\}$ be two sets of weights where $a_{i}$ and $b_{i}$ are associated with $p_{i}$ for $i=1, \ldots, n$. Then $A$ is said to majorize $B$ if for every point $p_{i}$ in $P$, the sum of the $a$ weights of all points $\left\{p_{j}: p_{j} \rightarrow p_{i}\right.$ or $\left.p_{j}=p_{i}\right\}$ is not less than the sum of the $b$ weights on the same set of points. With this viewpoint, it is natural to consider majorization on a set of points $P$ partially ordered by " $\rightarrow$ " (read "dominates"). For $P^{\prime} \subseteq P$ let $A\left(P^{\prime}\right)$ (or $B\left(P^{\prime}\right)$ ) denote the sum of the weights ( $b$ weights) of all the points $\left\{p_{j}: p_{j} \in P^{\prime}\right.$ or $p_{j} \rightarrow p_{i}$ for some $\left.p_{i} \in P^{\prime}\right\}$. Then we say that $A$ majorizes $B$ on $P$ if $A(P)=B(P)$ and for every $P^{\prime} \subset P, A\left(P^{\prime}\right) \geqslant B\left(P^{\prime}\right)$. Note that when $P$ is a linearly ordered set, then $A$ majorizing $B$ on $P$ is reduced to the definition of vector majorization.

In this paper we give a useful property which characterizes majorization on a partially ordered set. It turns out that to prove this property, we need to resort to some concepts and results in characteristic function games. Therefore we give a brief sketch of what we need from characteristic function games in \$2. Using the characterization property, we prove a theorem parallel to the Schur-Ostrowski theorem on set majorization. A similar theorem on vector majorization follows as a corollary to our theorem.

2. Some concepts and results in characteristic function games. For a set of players $N=\{1, \ldots, n\}$ a characteristic function $v(\cdot)$ is a real valued function assigning to each subset $S \subseteq N$ the number $v(S)$. This number may be thought of as describing the potential worth of the coalition $S$. The function $v(\cdot)$ completely determines the strategic possibilities of the game. A game is convex if its characteristic function satisfies $v(\phi)=0$ and

$$
v(S)+v(T)<v(S \cup T)+v(S \cap T)
$$

for any $S, T \subseteq N$.

The core of a characteristic function game is the set of solutions to the following set of equations

$$
\begin{aligned}
& \sum_{i \in S} x_{i} \geqslant v(S) \text { for } S \subset N, \\
& \sum_{i \in N} x_{i}=v(N) .
\end{aligned}
$$

The core can be described intuitively as the set of payoff vectors that leave no coalition in a position to improve the payoffs of all its members. A characteristic function game need not have a core. However, Shapley [6] proved the following.

THEOREM 2.1. The core exists for every convex game. 
It is quite unexpected that this theorem will be needed to prove a property of majorization on a partially ordered set.

3. The main theorems. Consider a given set of points $P=\left\{p_{1}, \ldots, p_{n}\right\}$ partially ordered by " $\rightarrow$ " and a set of weights $A$ associated with $P$. Let $p_{i}$ and $p_{j}$ be two points in $P$ such that $p_{i} \rightarrow p_{j}$. Then a flow from $a_{i}$ to $a_{j}$ is a transformation from $A$ to $A^{\prime}$ where

$$
\begin{aligned}
& a_{i}^{\prime}=a_{i}-\delta, \\
& a_{j}^{\prime}=a_{j}+\delta, \\
& a_{k}^{\prime}=a_{k} \quad \text { for } k \neq i, j,
\end{aligned}
$$

for some $\delta>0$.

THEOREM 3.1. Let $A$ and $B$ be two sets of weights associated with the partially ordered set $P$. Then $A$ majorizes $B$ on $P$ if and only if $A$ can be transformed into $B$ by a finite set of flows (in fact, at most $\left(\begin{array}{l}n \\ 2\end{array}\right)$ flows are needed).

Proof. (i) The "if" direction. If $A$ can be transformed into $A^{\prime}$ by a flow, then clearly $A$ majorizes $A^{\prime}$ on $P$. Since majorization on $P$ is transitive, $A$ majorizes $B$ on $P$.

(ii) The "only if" direction. Suppose $A$ majorizes $B$ on $P$; we show that there exists a finite set of flows transforming $A$ into $B$. We prove this by induction on the number of points in $P$. Let $q$ be a point of $P$ which is not dominated by any other point of $P$. If $q$ does not dominate any other point we ignore $q$ and prove Theorem 3.1 by induction. Otherwise let $q_{1}, \ldots, q_{j}$ be the points dominated by $q$, but not dominated by any other points dominated by $q$. If $a_{q}=b_{q}$, then again, we can ignore $q$ and prove Theorem 3.1 by induction. So we assume $a_{q}-b_{q}=\theta>0$. We now show that there exists a set of weights $A^{\prime}$ which can be obtained from $A$ by flowing the amount $\theta_{i}>0$ from $q$ to $q_{i}, i=1, \ldots, j$, such that $\Sigma_{i=1}^{j} \theta_{i}=\theta$ and $A^{\prime}$ majorizes $B$ on $P$. Once this has been proved, then by induction $A^{\prime}$ can be transformed into $B$ by a finite set of flows. Consequently, $A$ can be transformed into $B$ by a finite set of flows and Theorem 3.1 follows.

For $P^{\prime} \subseteq P$, define

$$
\bar{A}\left(P^{\prime}\right)=A\left(P^{\prime}\right)-\theta .
$$

Let $K$ be a subset of $J=(1, \ldots, j)$. Define

$$
v_{K}=\operatorname{Max}_{P_{K}}\left(B\left(P_{K}\right)-\bar{A}\left(P_{K}\right)\right)
$$

where $P_{K}$ is a subset of $P-\{q\}$ containing $K$ but not any element from $J-K$. Then $A^{\prime}$ majorizes $B$ if and only if

$$
\sum_{i \in K} \theta_{i}>v_{K} \text { for every } K \subseteq J
$$

We can define a characteristic function game on the set of players $J$ by treating $\left\{v_{K}: K \subseteq J\right\}$ as the characteristic function. Then 


$$
\sum_{i \in K} \theta_{i} \geqslant v_{K} \text { for every } K \subseteq J
$$

is equivalent to the statement that the core of the game exists. We now prove the core exists by showing that the game is convex.

Let $P_{K}^{0}$ and $P_{K^{\prime}}^{0}$ be two subsets of $J$ such that

$$
v_{K}=B\left(P_{K}^{0}\right)-\bar{A}\left(P_{K}^{0}\right)
$$

and

$$
v_{K^{\prime}}=B\left(P_{K^{\prime}}^{0}\right)-\bar{A}\left(P_{K^{\prime}}^{0}\right)
$$

Then

$$
\begin{aligned}
v_{K \cup K^{\prime}} \geqslant & B\left(P_{K}^{0} \cup P_{K^{\prime}}^{0}\right)-\bar{A}\left(P_{K}^{0} \cup P_{K^{\prime}}^{0}\right) \\
= & B\left(P_{K}^{0}\right)+B\left(P_{K^{\prime}}^{0}\right)-B\left(P_{K}^{0} \cap P_{K^{\prime}}^{0}\right) \\
& -\bar{A}\left(P_{K}^{0}\right)-\bar{A}\left(P_{K^{\prime}}^{0}\right)+\bar{A}\left(P_{K}^{0} \cap P_{K^{\prime}}^{0}\right) \\
= & v_{K}+v_{K^{\prime}}-\left(B\left(P_{K}^{0} \cap P_{K^{\prime}}^{0}\right)-\bar{A}\left(P_{K}^{0} \cap P_{K^{\prime}}^{0}\right)\right) \\
\geqslant & v_{K}+v_{K^{\prime}}-v_{K \cap K^{\prime}}
\end{aligned}
$$

Therefore the game is convex and so the core exists by Theorem 2.1.

COROLlary. Suppose $P$ is a linearly ordered set. Then a necessary and sufficient condition for $A$ majorizing $B$ is the existence of an $n \times n$ triangular matrix $M=\left\{m_{i j}\right\}$ such that $m_{i j}>0, m_{i j}=0$ for $i<j, \sum_{j=1}^{n} m_{i j}=1$ and $B=M A$ (by interpreting $m_{i j} a_{i}$ as the amount of flow from $p_{i}$ to $p_{j}$ ).

Note that this corollary is very similar to Theorem 46 of [2] which says that a necessary and sufficient condition for a set $A$ majorizing a set $B$ is the existence of a doubly stochastic matrix $M$ such that $B=M A$.

The following theorem is parallel to the Schur-Ostrowski theorem.

THEOREM 3.2. Let $f\left(x_{1}, \ldots, x_{n}\right)$ be a function defined over the domain $D$. Let $P=\left(p_{1}, \ldots, p_{n}\right)$ be a set of points partially ordered by " $\rightarrow$ ". Then

$$
f\left(a_{1}, \ldots, a_{n}\right)>f\left(b_{1}, \ldots, b_{n}\right)
$$

for all $A$ majorizing $B$ on $P$ if and only if $f$ is such that for every $i$ and $j, p_{i} \rightarrow p_{j}$ implies

$$
\frac{\partial f}{\partial x_{i}}>\frac{\partial f}{\partial x_{j}} \quad \text { over all } X \in D \text {. }
$$

Proof. The "only if" part is trivial. The "if" part can be proved as follows. If there is a flow transforming $A$ into $A^{\prime}$, then clearly, $f(A)>f\left(A^{\prime}\right)$. From Theorem 3.1, there exists a finite set of flows transforming $A$ into $B$. Therefore Theorem 3.2 follows. 
Corollary. Let $f\left(x_{1}, \ldots, x_{n}\right)$ be a function defined over domain $D$. Let $P=\left(p_{1}, \ldots, p_{n}\right)$ be a vector. Then

$$
f\left(a_{1}, \ldots, a_{n}\right) \geqslant f\left(b_{1}, \ldots, b_{n}\right)
$$

for all $A$ majorizing $B$ (in the vector sense) if and only if $f$ is such that

$$
\left(\frac{\partial f}{\partial x_{i}}-\frac{\partial f}{\partial x_{j}}\right)(i-j)>0 .
$$

4. Conclusions. In this paper we extend the classical concept of set majorization to the case where the set is partially ordered. We give a mathematical definition of this new concept which includes "vector majorization" as a special case. Let $P$ and $P^{\prime}$ be two partial orders on the same set such that $p_{i} \rightarrow p_{j}$ in $P$ implies $p_{i} \rightarrow p_{j}$ in $P^{\prime}$. Then surprisingly, it is not true that if $A$ majorizes $B$ on the set under $P^{\prime}$, then $A$ majorizes $B$ on the set under $P$ (nor conversely) as is clear from the following example:

EXAMPLE. Let $P=\left(p_{1} \rightarrow p_{2}, p_{1} \rightarrow p_{3}\right), \quad P^{\prime}=\left(p_{1} \rightarrow p_{2} \rightarrow p_{3}\right), A=\left(x_{1}=\right.$ $\left..5, x_{2}=.5, x_{3}=0\right), B=\left(y_{1}=.4, y_{2}=.3, y_{3}=.3\right)$. Then $A$ majorizes $B$ on $P^{\prime}$ but not on $P$.

We also prove a property which characterizes majorization on a partially ordered set. Quite unexpectedly, the proof relies on a theorem of Shapley on convex games. Furthermore, we prove a theorem which is parallel to the Schur-Ostrowski theorem in comparing two functions except that the set majorization condition is replaced by a condition relating to the new notion of majorization.

The author wishes to thank a referee for suggesting the corollary of Theorem 3.1.

\section{REFERENCES}

1. F. R. K. Chung and F. K. Hwang, On blocking probabilities on a class of linear graphs, Bell System Tech. J. 57 (1978), 2915-2925.

2. G. H. Hardy, J. E. Littlewood and G. Pólya, Inequalities, 2nd ed., Cambridge Univ. Press, New York, 1959.

3. F. K. Hwang, Generalized Huffman trees, SIAM J. Appl. Math. (to appear).

4. A. Ostrowski, Sur quelques applications des fonctions convexes et concaves au sens de I. Schur, J. Math. Pures Appl. 31 (1952), 253-292.

5. I. Schur, Uber ein Klasse von Mittelbildungen mit Anwendungen auf die Determinantentheorie, Sitzungsber. Berlin. Math. Ges. 22 (1923), 9-20.

6. L. S. Shapley, Cores of convex games, Internat. J. Game Theory 1 (1971), 11-26.

Bell Laboratories, Murray Hill, New Jersey 07974 